\title{
SPECTROSCOPIC BINARIES AMONG A COMPLETE SAMPLE OF HIPPARCOS M GIANTS
}

\author{
A. Jorissen, ${ }^{1,2}$ B. Famaey, ${ }^{1,3}$ M. Dedecker, ${ }^{1}$ D. Pourbaix,${ }^{1,2}$ M. Mayor, ${ }^{4}$ and S. Udry ${ }^{4}$ \\ RESUMEN
}

Presentamos el primer diagrama período-excentricidad para binarias entre las estrellas gigantes $\mathrm{M}$ de campo; encontramos una frecuencia de binarias espectroscópicas de por lo menos $8 \%$.

\section{ABSTRACT}

We present the first eccentricity-period diagram for binaries among nearby field $\mathrm{M}$ giants, where an observed frequency of at least $8 \%$ of spectroscopic binaries is found.

\section{Key Words: BINARIES: SPECTROSCOPIC - STARS: LATE-TYPE - STARS: STATISTICS}

\section{THE OBSERVING CAMPAIGNS}

The present work is a by-product of the large observing campaign aiming at obtaining radial velocities for Hipparcos survey stars later than about F (for a detailed description of this campaign, see Udry et al. 1997). Two measurements per star, spanning at least $1 \mathrm{y}$, were obtained in the 1990s on the CORAVEL spectrovelocimeters (Baranne, Mayor \& Poncet 1979). Northern $M$ giants were extracted from this large database on the basis of the spectral type provided by the Hipparcos catalogue and of the absolute magnitude $M_{H_{p}}<4$ computed from the Hipparcos parallax and $H p$ magnitude. Mira stars or supergiants of luminosity class I (when explicitly mentioned in the spectral classification) were not included in the sample. The target list thus contains 707 northern $\mathrm{M}$ giants (sample I in Table 1).

Every third star from this complete sample has received a denser coverage, with (at least) 4 instead of 2 measurements, to achieve a better binary detection rate (sample II in Table 1). Finally, stars from the restricted sample II suspected of being binaries (i.e., with a radial-velocity standard deviation $\sigma\left(V_{r}\right)>1 \mathrm{~km} \mathrm{~s}^{-1}$; Figure 1) have been monitored to derive orbital elements. A summary of the sample properties is presented in Table 1 .

The search for spectroscopic binaries (SBs) among $\mathrm{M}$ giants is made difficult by the bulk mass motion existing in the atmospheres of these stars (all $M$ giants are variable to some extent), since such mo-

\footnotetext{
${ }^{1}$ Institut d'Astronomie et d'Astrophysique, Université Libre de Bruxelles, Belgium

(dedecker,bfamaey,ajorisse,pourbaix@astro.ulb.ac.be).

${ }^{2}$ Research Associate FNRS, Belgium.

${ }^{3}$ Boursier FRIA, Belgium.

${ }^{4}$ Observatoire de Genève, Switzerland (michel.mayor,stephane.udry@obs.unige.ch.
}

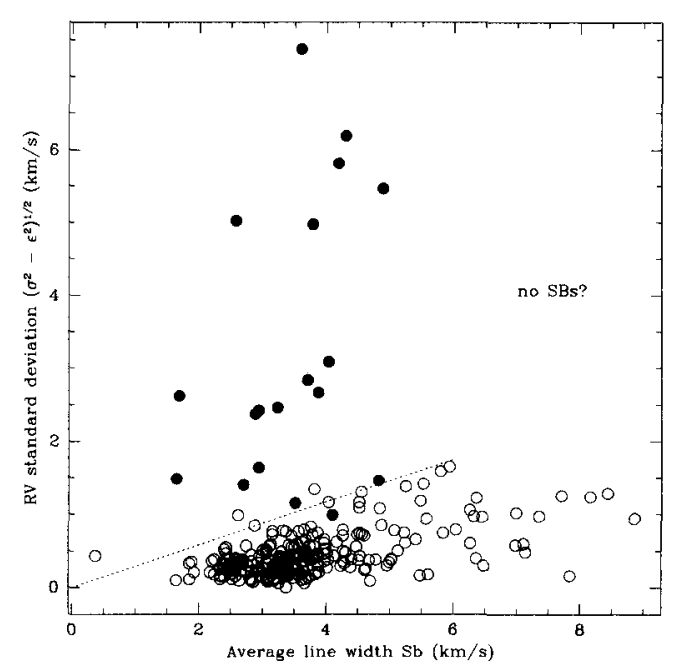

Fig. 1. The 'external' radial-velocity dispersion (i.e., the radial-velocity standard deviation $\sigma(V r)$ corrected for the instrumental error $\epsilon$ ) as a function of the average intrinsic line width $S b$, for sample II (see Table 1). The radial-velocity jitter increases with $S b$, and this evolution corresponds in fact to the transition towards $M$ giants of later spectral types (which have larger $S b$ values), and hence, of larger light variability. Confirmed SBs from the ELODIE campaign (sample III in Table 1) are denoted by black dots. The dashed line separates SBs (above) from radial-velocity jitter due to mass motion in the envelope (below). Note the lack of SBs among late $\mathrm{M}$ giants (with large $S b$ ).

tion triggers some intrinsic radial-velocity jitter (e.g., Udry et al. 1998; Hinkle et al. 2002). This intrinsic jitter amounts to nearly $2 \mathrm{~km} \mathrm{~s}^{-1}$ in the coolest $\mathrm{M}$ giants (Fig. 1), so that the detection of SBs cannot rely solely on a $\chi^{2}$ test comparing the radial-velocity standard deviation $\sigma(V r)$ to the instrumental error $\epsilon$. The parameter $S b$, measuring the average width 
TABLE 1

THE OBSERVING CAMPAIGNS OF HIPPARCOS M GIANTS, AND BINARY FREQUENCY

\begin{tabular}{cccc}
\hline Sample & I & II & III \\
\hline Selection & all M with $M_{H_{p}}<4, \delta>0^{\circ}$ & $\sim 1 / 3$ of I & $\sigma\left(V_{r}\right)>1 \mathrm{~km} \mathrm{~s}^{-1}$ from II \\
$N_{\text {star }}$ & 707 & 257 & 37 \\
$N_{\text {mas per star }}$ & 2 & $\geq 4$ & $\geq 5$ \\
Telescope & Swiss 1-m (OHP) & Swiss $1-\mathrm{m}(\mathrm{OHP})$ & $1.93-\mathrm{m}$ (OHP) \\
Spectrograph & CORAVEL & CORAVEL & ELODIE \\
Time span & $1991-1999$ & $1993-1995$ & $2000-.$. \\
\hline$N_{\text {bin }} / N_{\text {star }}$ & $41 / 707=5.8 \%$ & $20 / 257=7.8 \%$ & $20 \mathrm{SBs}, 14$ orbits \\
\hline
\end{tabular}

of spectral lines corrected for the instrumental width (see Jorissen \& Mayor 1988 for details), provides the supplementary criterion required to distinguish intrinsic jitter from orbital radial-velocity variations, since the radial-velocity jitter correlates with $S b$ in late-type stars, as revealed by Figure 1. This figure thus makes it possible to distinguish intrinsic radialvelocity jitter (for stars falling below the dashed line) from orbital variations (for stars falling above the dashed line).

\section{THE FREQUENCY OF SPECTROSCOPIC BINARIES}

The binary frequency derived from campaigns II and III (Table 1) amounts to $20 / 257$, or $7.8 \%$ (black dots on Fig. 1). The longest period found so far in the sample is $4367 \mathrm{~d}$ for HD 65275, but the recordholders are HD 192867 (Griffin 1990) and HD 115521 (a RV standard star; Udry et al. in preparation).

The above observed frequency is obviously a lower limit to the true binary frequency, since very longperiod or low-amplitude binaries may have $\sigma(V r) \leq$ $1 \mathrm{~km} \mathrm{~s}^{-1}$ in Fig. 1, and would thus have been missed by campaign III. To detect very long-period binaries among sample II, all stars are currently being remeasured, but results are not yet available.

The observed spectroscopic-binary frequency of about $8 \%$ for field $M$ giants appears significantly lower than the observed frequency of $23 \%$ for giants in open clusters (Mermilliod 2001).

\section{THE ECCENTRICITY-PERIOD DIAGRAM}

Figure 2 compares the position of $\mathrm{K}, \mathrm{M}$ and $\mathrm{Ba}$ giants in the eccentricity-period diagram. Quite interestingly, the region occupied by SBs moves to the lower right corner as one moves along the sequence KIII-MIII-Ba giants. This sequence of SBs involves stars with increasingly larger radii where circularization processes are operative at longer and longer periods (in the case of $\mathrm{Ba}$ giants, it is their white dwarf companions which reached very large radii while evolving all the way to the tip of the AGB).

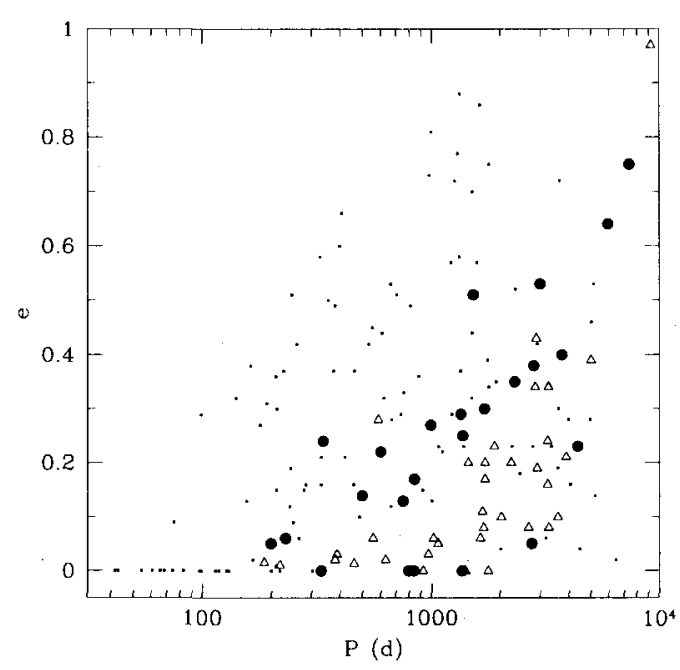

Fig. 2. The eccentricity-period diagram for SBs involving $M$ giants (black dots, from this work and collected from the literature, mostly from Hinkle et al. 2002 and references therein), $\mathrm{K}$ giants (small dots, from Mermilliod 1996), and strong Ba stars (Ba3-Ba5; open triangles, from Jorissen et al. 1998).

\section{REFERENCES}

Baranne, A., Mayor, M., \& Poncet, J. L. 1979, Vistas in Astronomy, 23, 279

Griffin, R. F. 1990, The Observatory, 110, 85

Hinkle, K. H., Lebzelter, T., Joyce, R. R., \& Fekel, F. C. 2002, AJ, 123, 1002

Jorissen, A., \& Mayor, M. 1988, A\&A, 198, 187

Jorissen, A., Van Eck, S., Mayor, M., \& Udry, S. 1998, $A \& A, 332,877$

Mermilliod, J.-C. 1996, in ASP Conf. Ser., 90, The Origins, Evolution, and Destinies of Binary Stars in Clusters, eds. E. Milone \& J.-C. Mermilliod (San Francisco: ASP), 95

Mermilliod, J.-C. 2001, in Astrophys. Space Sc. Lib., 264, The Influence of Binaries on Stellar Population Studies, ed. D. Vanbeveren (Dordrecht: Kluwer), 3

Udry, S. et al. 1997, in ESA-SP 402, "Hipparcos - Venice '97', eds. B. Battrick, M. A. C. Perryman, \& P. L. Bernacca (Noordwijk: ESA), 693

Udry, S., Jorissen, A., Mayor, M., \& Van Eck, S. 1998, A\&AS, 131, 25 